\section{Kidney Blood Pressure Research}

\title{
AF1q Contributes to Adriamycin-Induced Podocyte Injury by Activating Wnt/B- Catenin Signaling
}

\author{
Hongbo Zhang ${ }^{\mathrm{a}} \quad$ Rui Ren ${ }^{\mathrm{b}} \quad$ Juan Du ${ }^{\mathrm{a}} \quad$ Tingli Sun $^{\mathrm{a}} \quad$ Ping Wang $^{\mathrm{c}} \quad$ Ping Kang $^{\mathrm{a}}$ \\ aDepartment of Nephrology, Daqing Oil Field General Hospital, NO.9 Saertu District Daqing City, \\ Heilongjiang Province, ${ }^{b}$ Department of Hygiene Toxicology, School of Public Health, Harbin Medical \\ University, Harbin City, Heilongjiang Province, 'Department of Nutriology, Daqing Oil Field General \\ Hospital, NO.9 Saertu District Daqing City, Heilongjiang Province, China
}

\section{Key Words}

Podocytes injury • The ALL1-fused from the chromosome 1q (AF1q) • Wnt/ $\beta$-catenin signaling - Chronic kidney diseases (CKD) • Adriamycin (ADR)

\begin{abstract}
Background/Aims: Injury of podocytes plays an important role in decline of glomerular filtration and proteinuria. It is well-known that proteinuria is associated with numerous chronic kidney diseases (CKD). However, the underlying mechanism of podocyte injury remains unclear. Methods: We used reverse transcription-quantitative PCR (RT-qPCR) to compare the expression level of the ALL1-fused from the chromosome 1q (AF1q) gene in mice and mouse podocytes (MPC5) with or without Adriamycin (ADR) treatment. The effects of AF1q on Wnt/ $\beta$-catenin signaling were investigated by determining the expressions of desmin, snail, WT1, nephrin and E-cadherin using western blotting. Results: We found that AF1q expression was elevated in podocytes treated with ADR than untreated cells. AF1q overexpression directly led to podocytes injury with increased levels of desmin and snail. Luciferase activity of TOPflash reporter was significantly increased in cells with AF1q overexpression than wild type cells whereas deletion of T-cell-factor-7 (TCF7) eliminated this effect. Immunoprecipitation assay evidenced that AF1q interacted with TCF7 and promoted both transcriptional and translational expressions of TCF7. Overexpression of AF1q increased protein expression of $\beta$-catenin. However, in podocytes with deletion of TCF7, AF1q was not able to promote $\beta$-catenin expression. Conclusion: Our findings demonstrated that aberrant expression of AF1q may activate $\mathrm{Wnt} / \mathrm{\beta}$-catenin signaling and result in podocyte injury.

\section{Introduction}

Podocytes are highly differentiated perivascular cells developed from embryonic precursor mesenchymal cells [1]. In the kidney, they form an elaborate cytoarchitecture with




\section{Kidney Blood Pressure Research}

regularly spaced and interdigitated foot processes surrounding the glomerular capillaries, which plays an essential role in maintaining the integrity and function of the glomerular filtration barrier (GFB) [2]. Thus, injury of podocytes may result in defective glomerular filtration and is the main cause of proteinuria [3]. A number of chronic kidney diseases (CKD) at early stage exhibit proteinuria [4]. Additionally, podocyte injury has been evidenced as a key factor in the pathogenesis of proteinuria that occurs in majority of patients with CKD [4]. Mutations of several genes encoding proteins such as nephrin and podocin that are involved in the establishment of GFB leads to the development of proteinuria [5]. However, in clinical CKD cases, few patients are identified with these mutations and the mechanism of podocyte injury remains unclear.

Wnt gene family encodes secreted glycoproteins participating in a series of biological processes such as cell growth and differentiation. Canonical Wnt/ $\beta$-catenin signaling was first identified in Drosophila and is evolutionally conserved in all metazoans. It has a critical role in the development of kidney development. However, it is commonly silenced in adult kidney [6]. Recently, increasing studies have reported the correlation between Wnt/ $\beta$-catenin signaling and podocyte injury [7]. Nuclear translocation of $\beta$-catenin was enhanced by administration of puromycin to podocytes [8]. Wang $\mathrm{D}$ et al. demonstrated that Adriamycin (ADR) or transforming growth factor- $\beta$ (TGF- $\beta$ ) are involved in activation of Wnt/ $\beta$-catenin signaling and results in podocyte injury and proteinuria [9]. In fact, ADR nephropathy was established and has been long applied as an experimental model of kidney disease such as glomerulosclerosis $[10,11]$. Although ADR increases the level of reactive oxygen species and stimulates Wnt signaling, it has been demonstrated that the main contributions of ADR to kidney injury are mutations in mitochondrial DNA and decrease of it copy number [12]. Additionally, the Wilms' tumor suppressor gene (Wt1) that is limitedly expressed in the podocytes of adult kidney was reported to modulate Wnt/ $\beta$-catenin signaling and consequently cause podocyte dysfunction and apoptosis [13]. Therefore, a clear understanding of Wnt signaling may be essential for development of efficient treatment of CKD.

The ALL1-fused from the chromosome 1q (AF1q) gene is discovered from acute myeloid leukemia (AML) patients and restrictedly expressed in the differentiation of T-cells [14]. Although no studies have investigated the roles of AF1q in kidney, it was recently reported that AF1q could stimulate Wnt signaling by directly binding to T-cell-factor-7 (TCF7) [15]. In present study, we aimed to investigate whether AF1q participates in the pathogenesis of podocytes injury and its underlying mechanism.

\section{Materials and Methods}

\section{Mice}

Podocyte injury of BALB/c mice weighing 20-24 g and aged 6 weeks was induced by ADR as previously described [16]. Mice were treated with ADR for 3, 6 or 12 days and then sacrificed to obtain glomeruli $(n=5$ each group). For testing the effects of AF1q on kidney in vivo, mice were injected with vector or Flag-AF1q via tail vein using hydrodynamics-based procedure (1.8 ml per mouse) as previously stated [17]. After $24 \mathrm{~h}$, ADR (10.5 mg/kg Doxorubicin hydrochloride; Sigma, St. Louis, MO, USA) was administrated via intravenous injection. Sieving technique was used to isolate the glomeruli from kidney as previously described [18]. This study was carried out in strict accordance with the recommendations in the Guide for the Care and Use of Laboratory Animals of the National Institutes of Health. The protocol was approved by the Committee on the Ethics of Animal Experiments of Daqing Oil Field General Hospital.

\section{Cell and treatment}

Conditionally immortalized mouse podocytes (MPC5) were cultured in RPMI-1640 medium (Invitrogen, Carlsbad, CA, USA) supplemented with 10\% v/v fetal bovine serum (FBS, Gibco, Grand Island, $\mathrm{NY}$ ) at $33{ }^{\circ} \mathrm{C}$ and $5 \% \mathrm{CO}_{2}$ with $10 \mathrm{U} / \mathrm{ml}$ of interferon- $\gamma$ (BioSource, Waltham, MA) and differentiated at 


\section{Kidney Blood Pressure Research}

Zhang et al.: Aberrant Expression of AF1q in Podocyte Injury

$37{ }^{\circ} \mathrm{C}$ (without interferon- $\gamma$ ) supplemented with $10 \% \mathrm{v} / \mathrm{v}$ FBS. After differentiation for $10-14$ days, the podocytes were treated with ADR $(3 \mu \mathrm{g} / \mathrm{ml})$ for $6 \mathrm{~h}, 12 \mathrm{~h}$ and $24 \mathrm{~h}$. Flag-AF1q and shTCF7 were constructed for overexpression of AF1q and deletion of TCF7, respectively. Additionally, HEK-293T cell line (BIOLEAF, China) was cultured in Dulbecco's modified Eagle's medium (DMEM) supplied with 10\% v/v FBS (Sigma) and $1 \% \mathrm{v} / \mathrm{v}$ streptomycin and penicillin at $37{ }^{\circ} \mathrm{C}$ in a humidified incubator with $5 \% \mathrm{CO}_{2}$.

Reverse transcription-quantitative PCR (RT-qPCR)

RNA was extracted using TRIzol reagent (Ambion, Austin, TX, USA) according to the manufacturer's instructions. Reverse cDNA synthesis was performed using a cDNA synthesis kit (Promega, Madison, WI, USA) according to the instructions of the manufacturer. Primers used in RT-qPCR for detecting AF1q and TCF7 are as following: AF1q forward, 5'-TCAGATACAGCCACCTAC-3'; Reverse, 5'-TAGAGCAAGTCCATGTCG-3'; TCF7 forward 5'- TGCCTTGGACTCTGCTTGTG -3'; Reverse, 5'- CGTCTACTCCGGCTTCAATCTG -3'. Values were normalized to GAPDH. GAPDH forward, 5'-CCTCGTCCCGTAGACAAAATG-3'; reverse, 5'-TCTCCACTTTGCCACTGCAA-3'. The relative quantity of the products was calculated as follow: $\mathrm{RQ}=1 / \mathrm{E}^{\mathrm{Cq}}$

\section{Western blotting}

Cells were lysed by RIPA buffer (Thermo Fisher Scientific, Waltham, MA). Sodium dodecyl sulfatepolyacrylamide gel electrophoresis (SDS-PAGE) was used for separating proteins. Proteins were transferred onto a nitrocellulose membrane (Millipore, Billerica, MA, USA). The primary antibodies (1:1000 diluted in tris-buffered saline with $0.1 \%$ tween, TBST) were used to detect desmin (Sigma), snail (Sigma), WT1 (Sigma), nephrin (Sigma), E-cadherin (Sigma), tubulin (Sigma), Flag (Sigma), $\beta$-catenin (Abcam, Cambridge, MA), myc tag (Abcam), TCF7 (Abcam).

\section{Hematoxylin and eosin (HE) staining}

Renal sample was placed in 10\% neutral-buffered formalin fixative at room temperature for 12 hour and embedded in paraffin. Then, samples were stained by hematoxylin and eosin (HE).

\section{Luciferase assay}

The effect of AF1q on Wnt/ $\beta$-catenin signaling was detected using a TOPflash reporter plasmid with TCF binding site upstream of the thymidine kinase (TK) minimal promoter and luciferase open reading frame (Millipore, USA). For transfection, TOP-flash plasmid and an internal control reporter plasmid $(0.1 \mu \mathrm{g})$ Renilla reniformis luciferase driven under the TK promoter (pRL-TK; Promega, USA) were simultaneously transfected in cells. Luciferase activity was measured using a dual luciferase assay system kit (Promega, Madison, WI) according to the manufacturer's protocols and was observed by a Synergy H1 microplate reader (Biotek, Vermont, USA).

\section{Statistical analysis}

All data were presented as mean \pm SD (standard deviation). Statistical analysis of the data was performed using GraphPad Prism 5 software (GraphPad Software, Inc., La Jolla, CA, USA). Values of $\mathrm{p}<0.05$ were considered as statistically significant. All experiments were performed at least 3 times.

\section{Results}

AF1q expression is upregulated in injured podocytes

To test whether AF1q is related to ADR-induced kidney injury, BALB/C mice were treated with ADR $(10.5 \mathrm{mg} / \mathrm{kg})$ by a tail vein injection. Mice were sacrificed on day 3, 6 or 12 after ADR treatment and glomeruli were isolated to evaluate AF1q mRNA level. We found that the transcriptional level of AF1q in glomeruli was significantly elevated in mice with ADR treatment compared with it in untreated mice (Fig. 1A). We further validated this result in MPC5 cell line. As shown in Fig. 1B, both $1 \mu \mathrm{g} / \mathrm{ml}$ and $3 \mu \mathrm{g} / \mathrm{ml}$ ADR elicited remarkably increases of AF1q mRNA expression. When MPC5 cells were treated with $3 \mu \mathrm{g} / \mathrm{ml}$ ADR for 6 , 12 and 24 h, AF1q mRNA level was enhanced with duration of treatment whereas no change 


\section{Kidney \\ Blood Pressure Research}

was observed in untreated cells (Fig. 1C). To clarify the function of AF1q in ADR-induced kidney injury, we established a AF1q-knockdown MPC5 cell line which were then treated by ADR ( $3 \mu \mathrm{g} / \mathrm{ml})$ for $12 \mathrm{~h}$. We found that podocyte damage was not induced by ADR in the absence of AF1q (Fig. 2).

AF1q plays a role in podocyte injury and renal damage

To determine whether AF1q is related to podocyte injury, the protein levels of desmin and snail, two markers of podocyte injury, were compared between control and AF1q overexpressing cells. We observed that AF1q led to significant increases of protein expressions of desmin and snail in comparison with control (Fig. $3 \mathrm{~A})$. On the contrary, the expression levels of WT1, nephrin and E-cadherin as three podocyte functional markers were markedly repressed by AF1q. Then, HE staining results showed that mice with AF1q overexpression exhibited tubular dilation or deformation, and accompanied by glomerular hypertrophy in kidney compared to control mice (Fig. 3B), indicating that AF1q aggravated podocyte injury and renal damage.

The function of $A F 1 q$ on kidney is mediated by Wnt/ $\beta$-catenin signaling

We then investigated the signaling pathway involved in AF1q-induced changes in kidney. It was observed that TOPflash reporter activity (luciferase reporter containing 3 optimal TCF binding sites) in AF1q overexpressed cells was significantly higher than it in control cells (Fig. 4A). Protein level of $\beta$-catenin was comparably enhanced by AF1q (Fig. 4B), suggesting that Wnt/ $\beta$-catenin signaling may be involved in AF1q-induced kidney injury.

AF1q enhances TCF7 expression and interacts with TCF7 in vitro

It was reported that $\mathrm{AF} 1 \mathrm{q}$ enhanced Wnt signaling pathway

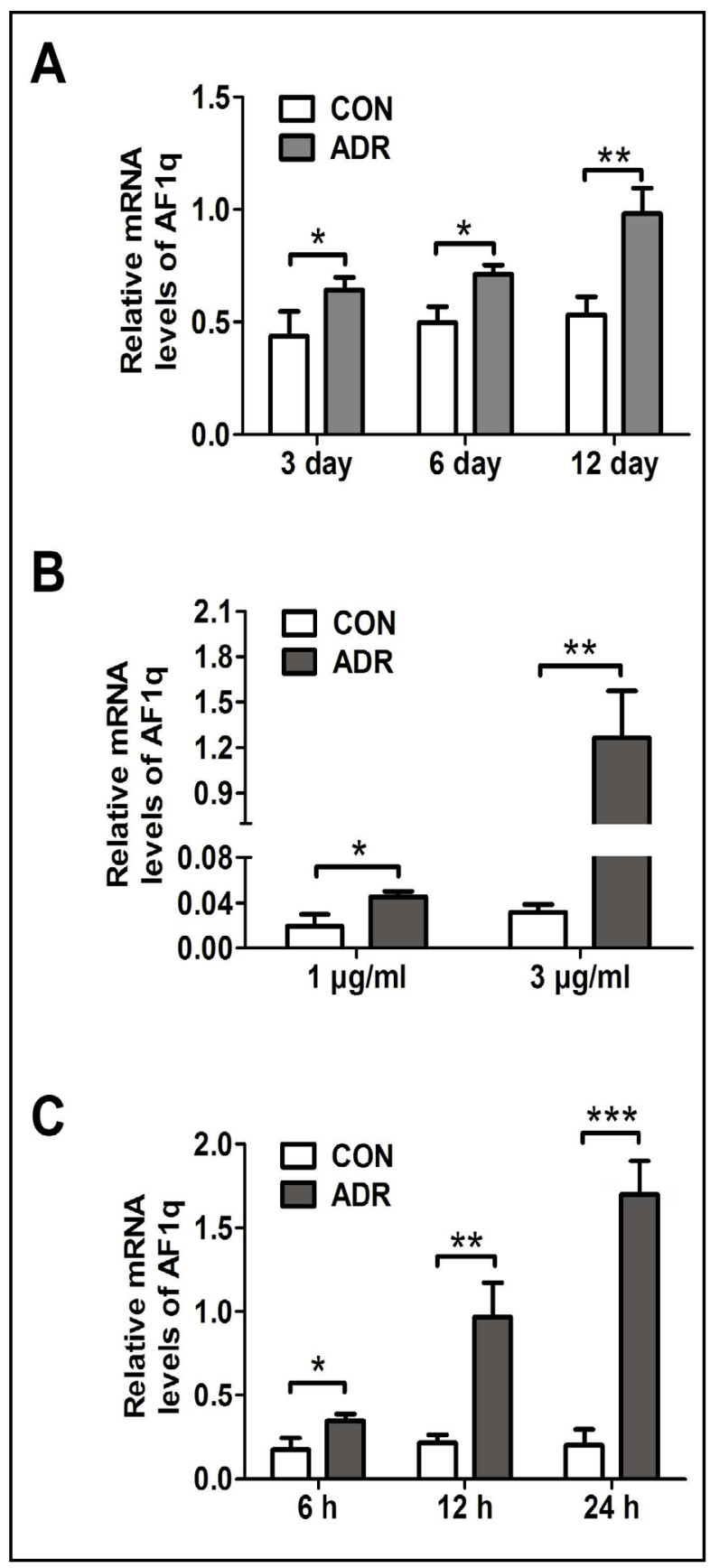

Fig. 1. AF1q is upregulated in injured podocytes. AF1q expression was evaluated by reverse transcriptionquantitative PCR (RT-qPCR) in the isolated glomeruli from $\mathrm{BALB} / \mathrm{c}$ mice treated with adriamycin (ADR; $10.5 \mathrm{mg} /$ $\mathrm{kg}$ ) for 3, 6 or 12 days. (B) AF1q expression was analyzed in MPC5 cells (mouse podocyte cell line 5) treated with different doses of ADR by RT-qPCR. (C) AF1q expression was evaluated in MPC5 cells treated with ADR $(3 \mu \mathrm{g} / \mathrm{ml})$ for $6 \mathrm{~h}, 12 \mathrm{~h}$ or $24 \mathrm{~h}$ by RT-qPCR. Data were presented as mean \pm SD. ${ }^{*} \mathrm{p}<0.05,{ }^{* *} \mathrm{p}<0.01$ and ${ }^{* * *} \mathrm{p}<0.001$. 


\section{Kidney Blood Pressure Research}

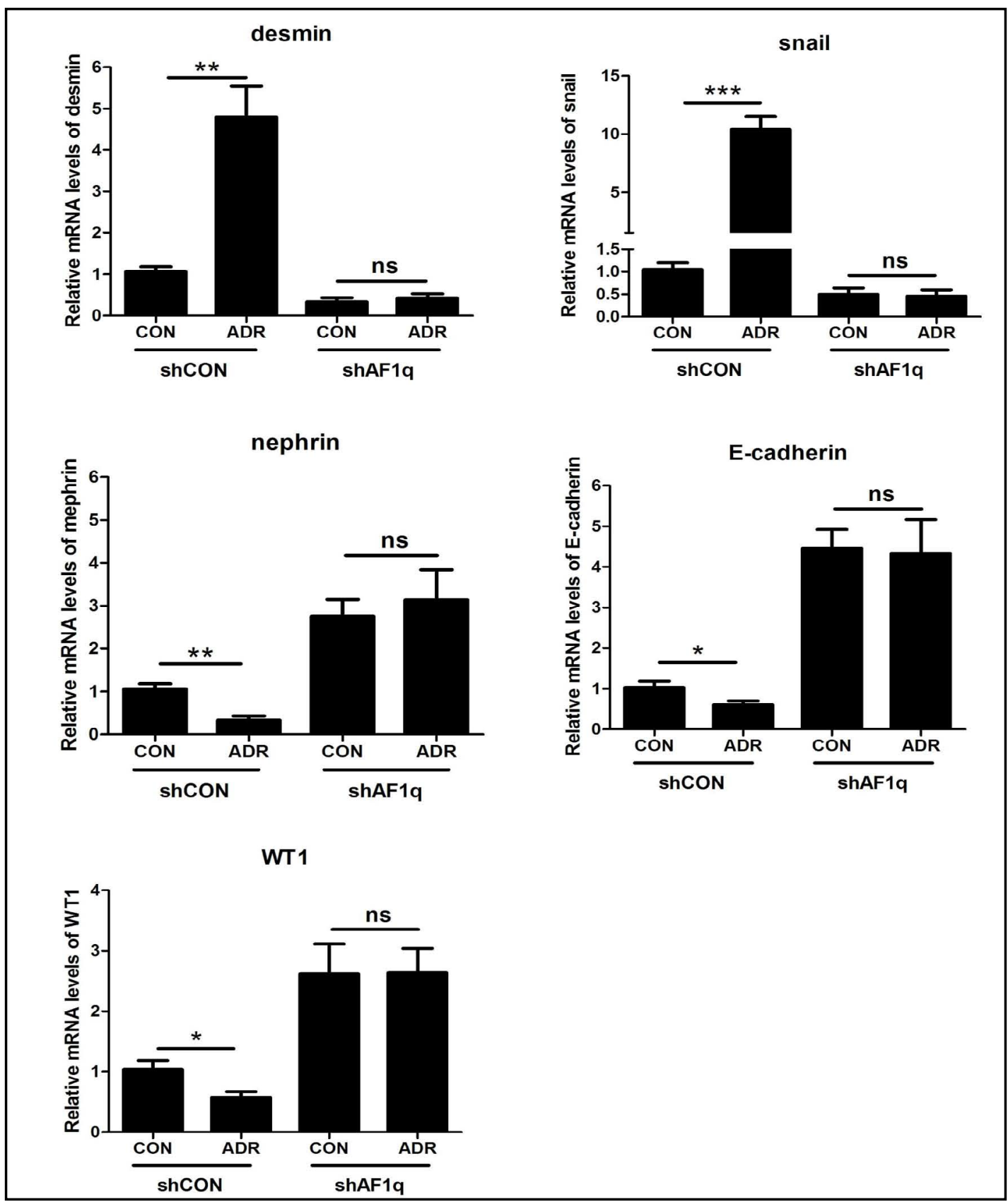

Fig. 2. ADR-mediated podocyte damage would not occur in the absence of AF1q. MPC5 cells transfected with shCON or shAF1q were treated with CON or ADR $(3 \mu \mathrm{g} / \mathrm{ml})$ for $12 \mathrm{~h}$. mRNA levels of desmin, snail, nephrin, E-cadherin or WT1 were evaluated by Realtime PCR.

by interacting with TCF7 to activate CD44 transcription [15]. We further confirmed the interactions between AF1q and TCF7. As shown in Fig. 5 A and B, myc-TCF7 was immunoprecipitated by Flag-AF1q using anti-Flag antibody and vice versa. Additionally, AF1q overexpression in 293T cells resulted in significant increase of TCF7 mRNA expression compared to control cells (Fig. 5C). 


\section{Kidney Blood Pressure Research}

Kidney Blood Press Res 2017;42:794-803

\begin{tabular}{|l|l|}
\hline DOI: $10.1159 / 000484329$ & (C) 2017 The Author(s). Published by S. Karger AG, Basel
\end{tabular}

Published online: October 25, 2017 www.karger.com/kbr
Fig. 3. AF1q promotes podocyte injury and renal damage. (A) MPC5 cells transfected with vector or Flag-AF1q for $48 \mathrm{~h}$ were lysed and immunoblotted with antibodies against desmin, snail, nephrin, E-cadherin and WT1 as well as Tubulin as indicated. Relative protein expression levels of desmin, snail, nephrin, E-cadherin and WT1 were normalized to Tubulin. (B) HE staining analysis of renal pathological changes in mice with the administration of vector or Flag-AF1q. Data were presented as mean \pm SD. ${ }^{*} \mathrm{p}<0.05,{ }^{* *} \mathrm{p}<0.01$.

Knockdown of TCF7 eliminates the increase of $\beta$-catenin induced by $A F 1 q$

To further confirm the underlying mechanism of the effects of AF1q on Wnt signaling pathway, we tested the TOPflash reporter activity in TCF7 knockdown MPC5 cells. As expected, in TCF7 knockdown cells, no significant difference was observed between cells with or without AF1q transfection (Fig. 6A). However, AF1q transfection led to a significantly higher level of TOPflash reporter activity than un-transfected cells in control cells without deletion of TCF7. Moreover, AF1 overexpression also increased protein expression of $\beta$-catenin whereas this effect was eliminated after TCF7 knockdown (Fig. 6B).

\section{Discussion}

Under pathophysiologic conditions, theresponses ofpodocytes to consistent stressors such as glomerular hyperfiltration and hypertension may comprise a spectrum of changes including hypertrophy, detachment, dedifferentiation, mesenchymal transition, mitotic catastrophe, and apoptosis [19]. Such changes may elicit dysfunction of podocytes and proteinuria. Thus, alleviation of these symptoms is essential for the treatment and several medicines have been found. Albuminuria was reported to be reduced by olmesartan through the inhibitory role of olmesatan in

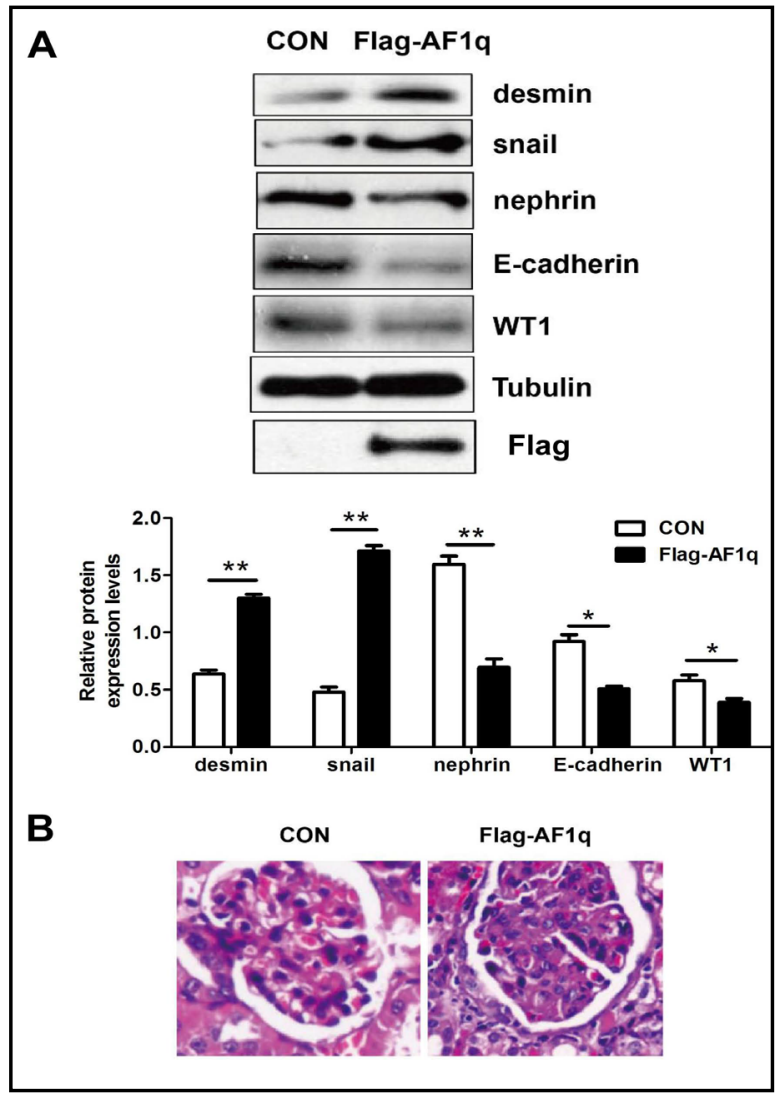

A

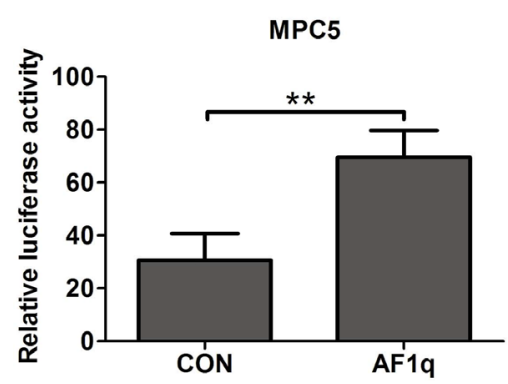

B

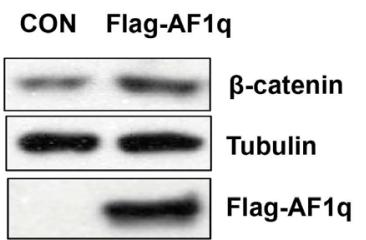

Fig. 4. $A F 1 q$ promotes the activation of $\mathrm{Wnt} / \beta$-catenin signaling. (A) Luciferase activity of TOPflash reporter was evaluated in MPC5 cells with or without AF1q overexpression by a dual-luciferase assay. (B) MPC5 cells transfected with vector or Flag- AF1q for $48 \mathrm{~h}$ were lysed and immunoblotted with unphosphorylated $\beta$ - catenin and Tubulin. Data were presented as mean \pm SD. ${ }^{* *} \mathrm{p}<0.01$. 


\section{Kidney Blood Pressure Research}

Fig. 5. AF1q enhances TCF7 expression and interacts with TCF7 in vitro. (A) HEK293T cells were transfected with Flag-AF1q or/and Myc-TCF7 plasmids and were lysed. Cell lysis was immunoprecipiated by Flag-AF1q with anti-Flag antibody, then western blotting was performed to evaluate Myc-TCF7 with antiMyc antibody. (B) HEK293T cells were transfected with Myc-TCF7 and vector or Flag-AF1q and then immunoblotted with antiMyc antibody. (C) RT-qPCR was performed to evaluate mRNA levels of TCF7 in HEK293T cells transfected with vector or Flag-AF1q. Data were presented as mean \pm SD. ${ }^{* * *} \mathrm{p}<0.001$.

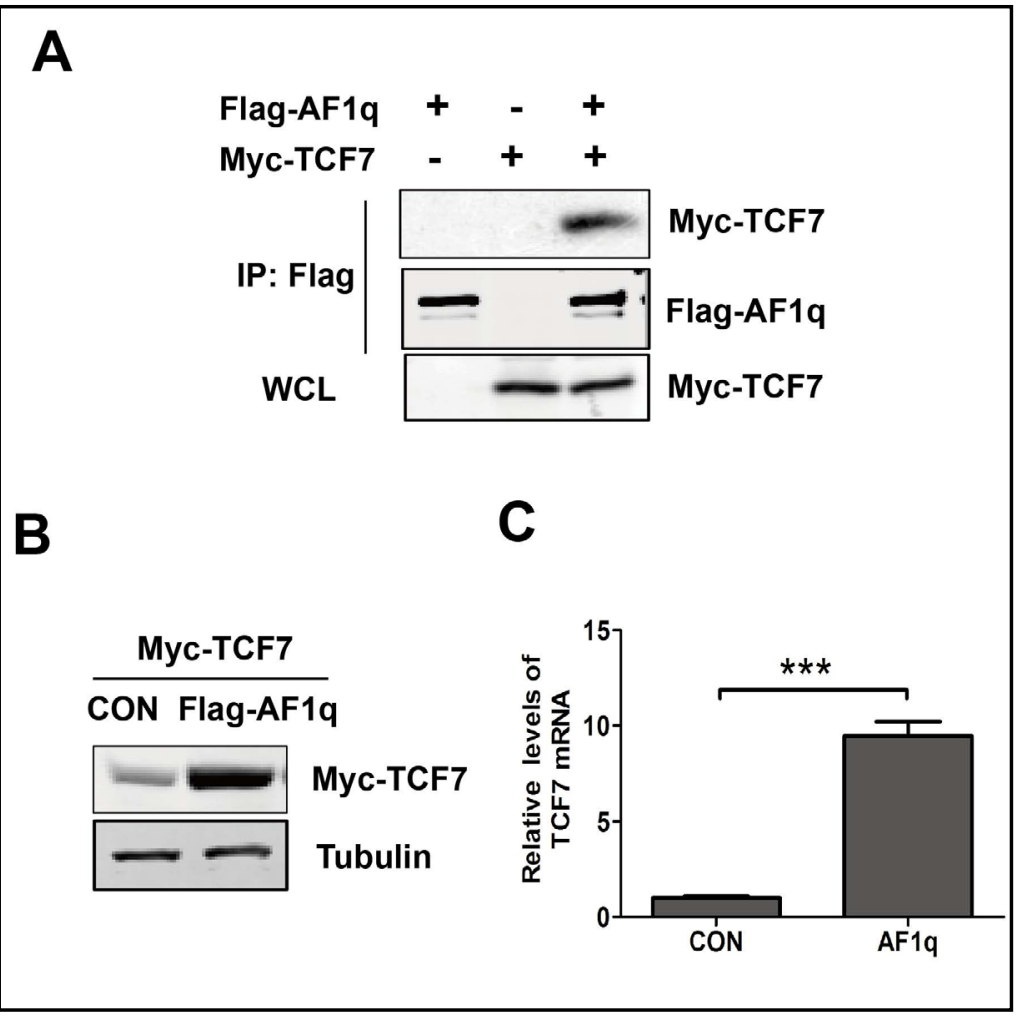

Fig. 6. Knockdown of TCF7 eliminates the increase of $\beta$-catenin induced by AF1q. (A) Luciferase activity of TOPflash reporter was evaluated in control (CON) and AF1q overexpressing (AF1q) MPC5 cells with (shTCF7) or without (shCON) knockdown of TCF7 by a dual-luciferase assays. (B) MPC5 cells transfected with shTCF7, vector, or FlagAF1q for $48 \mathrm{~h}$ were lysed and immunoblotted with unphosphorylated $\beta$-catenin and Tubulin. $\beta$-catenin expression was normalized to Tubulin. The relative

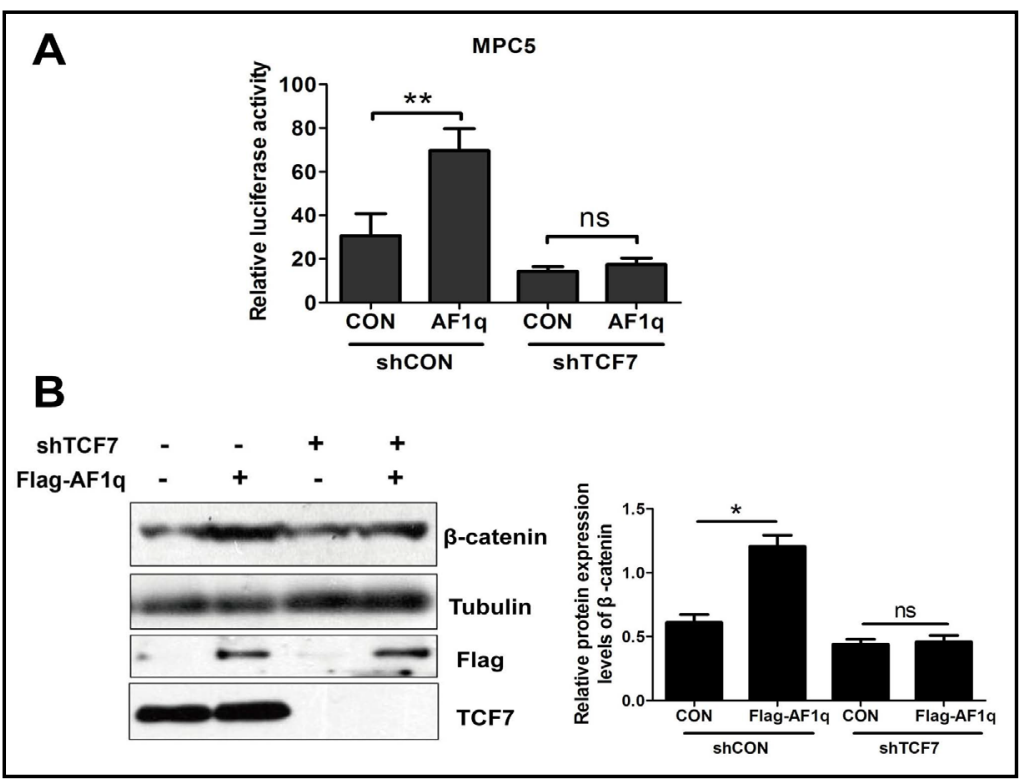
expressions of these proteins were also shown. Data were presented as mean \pm SD. ${ }^{*} \mathrm{p}<0.05,{ }^{* *} \mathrm{p}<0.01$. n.s indicates no statistical difference.

podocyte apoptosis [20]. Sinomenine was reported to repress autophagy of podocyte, which could prevent podocyte depletion [21]. However, new medicines are necessary for efficient treatment and detailed mechanism of podocytes injury needs to be revealed. Proteinuria is not only a marker for the progression of kidney injury but also mediates subsequent inflammatory responses. In adults, Wnt expression and signaling are normally silenced in the kidney. Sustained activation of Wnt signaling is critical in the pathogenesis of podocyte 


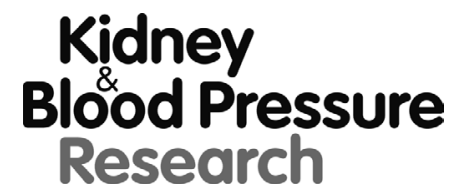

Kidney Blood Press Res 2017;42:794-803

\begin{tabular}{|l|l|l|}
\hline DOI: $10.1159 / 000484329$ & (c) 2017 The Author(s). Published by S. Karger AG, Basel \\
\hline
\end{tabular}

Published online: October 25, 2017

www.karger.com/kb

801

Zhang et al.: Aberrant Expression of AF1q in Podocyte Injury

dysfunction and proteinuria. Thus, understanding the mechanism of activation of Wnt signaling in podocytes is necessary.

Here, we found that transcriptional levels of AF1q were significantly higher in the glomeruli from ADR-treated mice than untreated mice. In vitro evidence in MPC5 cells also supported this observation, suggesting that AF1q may play a role in podocyte injury. By testing several markers of podocyte injury and function including desmin, snail, WT1, nephrin and E-cadherin, we demonstrated that high level of AF1q is associated with podocyte injury and dysfunction. Wnt/ $\beta$-Catenin Signaling was reported to induce snail and repress nephrin expressions, indicating an adverse effect of Wht signaling on podocyte function [22]. Recently, Park J et al. reported AF1q promotes metastasis of breast cancer via specifically binding to TCF7 in the Wnt signaling [15]. Based on the important role of Wnt in kidney, we investigated whether AF1q regulates Wnt signaling. We found that AF1q promoted the protein expression of $\beta$-catenin and interacted with TCF7. However, AF1q failed to enhance luciferase activity of TOPflash reporter and protein expression of $\beta$-catenin in TCF7 deleted cells. It is implied that AF1q regulates Wnt signaling via specifically binding to TCF7.

There are two Wnt/ $\beta$-catenin signaling pathways, canonical and noncanonical signaling. In previous studies, activation of $\beta$-catenin has been observed in podocytes of various proteinuric CKD [23]. Thus, under CKD condition, it appears that Wnt signaling is mainly activated by canonical pathway, which is dependent on $\beta$-catenin. Briefly, after binding of Wnt family to their receptor Frizzled and coreceptors LRP5/6, several events occur in downstream signaling involving a number of proteins including Disheveled, axin, and glycogen synthase kinase- $3 \beta$. Eventually these events stabilize $\beta$-catenin via dephosphorylation of $\beta$-catenin and translocate it to nuclei where it binds to TCF to activate transcriptions of Wnt target genes. In our results, overexpression of AF1q also elicited elevated expression of $\beta$-catenin. However, this effect may be indirect because we found that AF1q specifically binds to TCF7. It was demonstrated that AF1q promotes transcriptional rates through increasing the formation of transcriptional complex with lymphoid enhancer-binding factor (LEF) and $\beta$-catenin [15]. Additionally, we found that TCF7 transcriptional level was also increased by AF1q. Therefore, it is possible that AF1q promotes the formation of complex with TCF7 and also $\beta$-catenin, which in turn enhances TCF7 transcription. It was reported that inhibition of Wnt/ $\beta$-catenin signaling ameliorates podocyte dysfunction, proteinuria, and kidney injury after using paricalcitol (19-nor-1, 25-hydroxy-vitamin D2) [24].

The AF1q gene was originally identified in leukemia cells as a fusion partner for MLL protein and is highly expressed in leukemic and immature hematopoietic cells [14]. However, in normal hematopoietic tissue, its expression is mainly restricted in thymus. Several other tissues such as colon, small intestine, and ovary showed AF1q expression. We identified that the expression of AF1q was dramatically elevated in glomeruli from mice treated with ADR. The interaction between AF1q and Notch signaling was reported in the commitment intrathymic projenitros toward the T-cell lineage [25]. In recent years, the role of AF1q in neuronal cells has been confirmed by identification of AF1q spatiotemporal expression in brain and the ability of AF1q in transforming human embryonic kidney (HEK) cells into neuron-like cells $[26,27]$. Although the biological function of AF1q has not been completely characterized, there are increasing evidence showed a potential proto-oncogenic function of AF1q in numerous tumors such as breast cancer and testicular germ cell tumors [2830]. Based on these previous studies and the findings in our results, it is possible that there is a potential correlation between kidney disease and cancers because high expression of AF1q may both elicit cancers and kidney diseases. However, it also depends on the sites of aberrant AF1q expression. In 2013, Malkawi A et al. performed a retrospective study and demonstrated that glomerular filtration rate is related to survival of patients with AML [31]. It is hard to explain the reason for this observation but AF1q may affect the function of GFB and sequentially influence glomerular filtration rate. It is well known that acute renal failure is a common complication of patients with cancers by either the disease itself or treatment [32-34]. Additionally, CKD was reported to be a risk factor for lung and urinary tract cancers 


\section{Kidney Blood Pressure Research}

Zhang et al.: Aberrant Expression of AF1q in Podocyte Injury

in older people [35]. Women with CKD was shown to have a higher frequency of breast calcification than women without CKD [36]. However, it is necessary to obtain a deeper insight into the roles of AF1q in CKD, which may provide a therapeutic method for treatment of CKD or cancers.

\section{Conclusion}

We identified that AF1q expression was elevated in podocytes treated with ADR. AF1q overexpression directly led to podocytes injury with elevated levels of desmin and snail. The function of AF1q was dependent on its interaction withTCF7.

\section{Disclosure Statement}

The authors declare that there is no Disclosure Statements.

\section{References}

1 Reiser J, Altintas MM: Podocytes. F1000Research 2016;5

- Pavenstadt H, Kriz W, Kretzler M: Cell biology of the glomerular podocyte. Physiol Rev 2003;83:253-307.

3 Nagata M: Podocyte injury and its consequences. Kidney Int 2016;89:1221-1230.

4 Reiser J, Sever S: Podocyte biology and pathogenesis of kidney disease. Annu Rev Med 2013;64:357-366.

5 Grahammer F, Schell C, Huber TB: Molecular understanding of the slit diaphragm. Pediatr Nephrol 2013;28:1957-1962.

6 Iglesias DM, Hueber PA, Chu L, Campbell R, Patenaude AM, Dziarmaga AJ, Quinlan J, Mohamed O, Dufort D, Goodyer PR: Canonical wnt signaling during kidney development. Am J Physiol-Renal 2007;293:F494-F500.

7 Dai CS, Stolz DB, Kiss LP, Monga SP, Holzman LB, Liu YH: Wnt/beta-catenin signaling promotes podocyte dysfunction and albuminuria. J Am Soc Nephrol 2009;20:1997-2008.

-8 Teixeira VDC, Blattner SM, Li M, Anders HJ, Cohen CD, Edenhofer I, Calvaresi N, Merkle M, Rastaldi MP, Kretzler M: Functional consequences of integrin-linked kinase activation in podocyte damage. Kidney Int 2005;67:514-523.

-9 Wang D, Dai C, Li Y, Liu Y: Canonical wnt/beta-catenin signaling mediates transforming growth factorbeta1-driven podocyte injury and proteinuria. Kidney Int 2011;80:1159-1169.

10 Rossmann P, Matousovic K, Bohdanecka M: Experimental adriamycin nephropathy. Fine structure, morphometry, glomerular polyanion, and cell membrane antigens. J Pathol 1993;169:99-108.

$>11$ Kato H, Gruenwald A, Suh JH, Miner JH, Barisoni-Thomas L, Taketo MM, Faul C, Millar SE, Holzman LB, Susztak K: Wnt/beta-catenin pathway in podocytes integrates cell adhesion, differentiation, and survival. J Biol Chem 2011;286:26003-26015.

-12 Papeta N, Zheng Z, Schon EA, Brosel S, Altintas MM, Nasr SH, Reiser J, D’Agati VD, Gharavi AG: Prkdc participates in mitochondrial genome maintenance and prevents adriamycin-induced nephropathy in mice. J Clin Invest 2010;120:4055-4064.

13 Jing Z, Wei-jie Y, Yi-Feng ZG: Down-regulation of wt1 activates wnt/beta-catenin signaling through modulating endocytic route of lrp6 in podocyte dysfunction in vitro. Cell Signal 2015;27:1772-1780.

$\checkmark 14$ Tse W, Zhu W, Chen HS, Cohen A: A novel gene, af1q, fused to mll in $\mathrm{t}(1 ; 11)$ (q21;q23), is specifically expressed in leukemic and immature hematopoietic cells. Blood 1995;85:650-656.

15 Park J, Schlederer M, Schreiber M, Ice R, Merkel O, Bilban M, Hofbauer S, Kim S, Addison J, Zou J, Ji CY, Bunting ST, Wang ZQ, Shoham M, Huang G, Bago-Horvath Z, Gibson LF, Rojanasakul Y, Remick S, Ivanov A, Pugacheva E, Bunting KD, Moriggl R, Kenner L, Tse W: Af1q is a novel tcf 7 co-factor which activates cd44 and promotes breast cancer metastasis. Oncotarget 2015;6:20697-20710.

16 Fogo AB: Animal models of fsgs: Lessons for pathogenesis and treatment. Semin Nephrol 2003;23:161-171. 


\section{Kidney \\ Bloód Pressure Research}

\begin{tabular}{|c|c|}
\hline od Press Res 20 & $94-803$ \\
\hline $\begin{array}{l}\text { DOI: } 10.1159 / 000484329 \\
\text { Published online: October 25, } 2017\end{array}$ & $\begin{array}{l}\text { (c) } 2017 \text { The Author(s). Published by S. Karger AG, Basel } \\
\text { www.karger.com/kbr }\end{array}$ \\
\hline
\end{tabular}

Zhang et al.: Aberrant Expression of AF1q in Podocyte Injury

17 Liu F, Song YK, Liu D: Hydrodynamics-based transfection in animals by systemic administration of plasmid DNA. Gene Ther 1999;6:1258-1266.

18 Ni L, Saleem M, Mathieson PW: Podocyte culture: Tricks of the trade. Nephrology 2012;17:525-531.

19 Wiggins RC: The spectrum of podocytopathies: A unifying view of glomerular diseases. Kidney Int 2007;71:1205-1214.

20 Gu JH, Yang M, Qi N, Mei SQ, Chen JJ, Song SW, Jing Y, Chen MH, He LL, Sun LJ, Hu HM, Li L, Wuthrich RP, Wu M, Mei CL: Olmesartan prevents microalbuminuria in $\mathrm{db} / \mathrm{db}$ diabetic mice through inhibition of angiotensin ii/p38/sirt1-induced podocyte apoptosis. Kidney Blood Press R 2016;41:848-864.

21 Wang WL, Cai J, Tang S, Zhang Y, Gao XJ, Xie LJ, Mou ZR, Wu YZ, Wang L, Zhang JB: Sinomenine attenuates angiotensin II-induced autophagy via inhibition of $\mathrm{p} 47$-phox translocation to the membrane and influences reactive oxygen species generation in podocytes. Kidney Blood Press R 2016;41:158-167.

22 Dai C, Stolz DB, Kiss LP, Monga SP, Holzman LB, Liu Y: Wnt/beta-catenin signaling promotes podocyte dysfunction and albuminuria. J Am Soc Nephrol 2009;20:1997-2008.

23 Zhou L, Liu Y: Wnt/beta-catenin signalling and podocyte dysfunction in proteinuric kidney disease. Nat Rev Nephrol 2015;11:535-545.

24 He W, Kang YS, Dai C, Liu Y: Blockade of wnt/beta-catenin signaling by paricalcitol ameliorates proteinuria and kidney injury. J Am Soc Nephrol 2011;22:90-103.

25 Parcelier A, Maharzi N, Delord M, Robledo-Sarmiento M, Nelson E, Belakhdar-Mekid H, Pla M, Kuranda K, Parietti V, Goodhardt M, Legrand N, Bernstein ID, Gluckman JC, Sigaux F, Canque B: Af1q/mllt11 regulates the emergence of human prothymocytes through cooperative interaction with the notch signaling pathway. Blood 2011;118:1784-1796.

-26 Lin HJ, Shaffer KM, Sun ZR, Jay G, He WW, Ma W: Af1q, a differentially expressed gene during neuronal differentiation, transforms hek cells into neuron-like cells. Mol Brain Res 2004;131:126-130.

27 Yamada M, Clark J, Iulianella A: Mllt11/af1q is differentially expressed in maturing neurons during development. Gene Expr Patterns 2014;15:80-87.

28 Li DQ, Hou YF, Wu J, Chen Y, Lu JS, Di GH, Ou ZL, Shen ZZ, Ding J, Shao ZM: Gene expression profile analysis of an isogenic tumour metastasis model reveals a functional role for oncogene af1q in breast cancer metastasis. Eur J Cancer 2006;42:3274-3286.

29 Skotheim RI, Autio R, Lind GE, Kraggerud SM, Andrews PW, Monni O, Kallioniemi O, Lothe RA: Novel genomic aberrations in testicular germ cell tumors by array-cgh, and associated gene expression changes. Cell Oncol 2006;28:315-326.

30 Chang XZ, Li DQ, Hou YF, Wu J, Lu JS, Di GH, Jin W, Ou ZL, Shen ZZ, Shao ZM: Identification of the functional role of af1q in the progression of breast cancer. Breast Cancer Res Tr 2008;111:65-78.

31 Anand A, Al-Ameri A, Abdelfatah M, Kanaan Z, Haller N: Outcome of patients with acute myeloid leukemia/ high risk mds according to the kidney function. Blood 2013;122:5007-5007.

-32 Lameire NH, Flombaum CD, Moreau D, Ronco C: Acute renal failure in cancer patients. Ann Med 2005;37:13-25.

-33 Canet E, Zafrani L, Lambert J, Thieblemont C, Galicier L, Schnell D, Raffoux E, Lengline E, Chevret S, Darmon M, Azoulay E: Acute kidney injury in patients with newly diagnosed high-grade hematological malignancies: Impact on remission and survival. Plos One 2013;8:e55870.

34 Lameire N, Vanholder R, Van Biesen W, Benoit D: Acute kidney injury in critically ill cancer patients: An update. Crit Care 2016;20:209.

35 Wong G, Hayen A, Chapman JR, Webster AC, Wang JJ, Mitchell P, Craig JC: Association of ckd and cancer risk in older people. J Am Soc Nephrol 2009;20:1341-1350.

-36 Castellanos MR, Paramanathan K, El-Sayegh S, Forte F, Buchbinder S, Kleiner M: Breast cancer screening in women with chronic kidney disease: The unrecognized effects of metastatic soft-tissue calcification. Nat Clin Pract Nephr 2008;4:337-341. 